\title{
LA INFLUENCIA DE LA MÚSICA ÁRABE EN LA ESPAÑOLA DESDE MEDIADOS DEL S. XIX HASTA LOS AÑOS 30
}

Por

ENEIDA GARCIA GARIJO

\section{Introducción histórica}

En España, la Guerra de la Independencia fue la dolorosa culminación de una larga serie de conflictos tanto internos como externos. La situación no mejoró bajo el reinado de Fernando VII y debidos a su falta de habilidad política, a su muerte se desembocó en las Guerras Carlistas. Tampoco la Regencia y el reinado de Isabel II se caracterizaron por su tranquilidad. A continuación el gobierno provisional del general Serrano, el breve reinado de Amadeo, y la también breve República, para finalizar el siglo con el desastre de la pérdida de Cuba, Puerto Rico y las Filipinas, con lo que ello supuso en pérdida de vidas humanas y prestigio internacional.

Al iniciarse el siglo $X X$, las circunstancias de la vida española estaban delimitadas. La Restauración alfonsina y el sistema canovista de régimen rotatorio de gobierno, permitieron el asentamiento de una burguesía que, aunque solo era una débil imitación de la europea, llenaba un espectro social que hasta entonces no acababa de fijarse. Durante la minoría de Alfonso XIII y tras la pérdida de los últimos restos del imperio español, se produce un aldabonazo en la conciencia de los intelectuales principalmente, y la paz que existía -al menos superficialmente-, comienza a mostrar su fragilidad y a amenazar la estabilidad de un país que iba creando precariamente su industrialización, y arrastrando los problemas sociales que ello implicaba (R. Carr, 1983, pag. 74).

La neutralidad española en la I Guerra Mundial retuvo las tensiones y permitió una efímera euforia económica basada en la privilegiada posición de país 
neutral. Ello duraría poco y se vería barrido por la complicación de la aventura africana. Cuestiones que la Dictadura de Primo de Rivera no solucionó. Por ello, la Caida del dictador y la imposibilidad de seguir de sus sucesores, nacía bien previsible el futuro de la institución monárquica. Pero la proclamación de la Republica en 1931, alborozadamente acogida por la mayoría, se vió pronto que no era suficiente para solucionar la tensión nacional que se venía arrastrando desde el siglo anterior. Durante la mayor parte de la República se vivió un ambiente de pre-guerra que nacía presagiar cual sería su desenlace, plasmado en la rebelión militar de 1936.

\section{Conexión musical}

En este marco político y social que hemos resumido brevemente, se desarrolla el movimiento intelectual español, siendo la música una parte -aunque no excesivamente significativa- de ese conjunto. El atraso, que en líneas generales, padece la música en España durante el siglo XIX, ha sido objeto de grandes lamentaciones pero muy pocas veces se ha intentado explicar. Generalmente se ha achacado al continuo estado de guerra desde la invasión napoleónica, pasando por la guerra contra Marruecos hasta el desastre del 96 y Guerra Civil (C. Gómez, 1982, pag. 14).

Todos los motivos políticos y bélicos, ejercieron una clara acción negativa en la producción musical española, pero no son una explicación total. Es cierto que la guerra no suele ser un período floreciente para las artes, pero hay que matizar, según que arte. La guerra en ocasiones, puede ser un motivo de inspiración importante en pintura o en literatura, pero no lo es en la música. Por regla general, el acento guerrero que enciende himnos patrióticos, no suele inspirar a la gran creación sonora sino cuando se toma como motivo indirecto.

Pero no podemos achacar solo a la guerra y a la inestabilidad social, el atraso que durante tanto tiempo sufrió la musica en nuestro país y también la falta de hombres capacitados para realizarla. Quizá, el problema haya que buscarlo en la sociedad, incapaz de dar una respuesta adecuada a determinadas individualidades importantes.

Musicalmente, el siglo XIX español se puede definir como menor $y$ de carácter doméstico, pero forma parte del panorama cultural, aunque en muchas ocasiones haya sido relegado de este. Es cierto que la actividad musical a lo largo de todo el siglo es de segundo orden en líneas generales, y en ocasiones llega a aparecer, solo como un adorno en la educación. Pese a todo, la segunda mitad del siglo XIX ve florecer gran número de Sociedades Filarmónicas como la de Gran Canarias, Málaga (1869), Bilbao (1896) etc... En 1865 se crea la Sociedad de Conciertos de Madrid, base de la existencia musical de la ciudad entonces precaria, y de las Orquestas Sinfónicas y Filarmónica. La Real Academia de Bellas Artes de San Fernando, fundada por Felipe $\checkmark$ en 1744, no tuvo una sección de música hasta mayo de 1873 (A. FernándezCid, 1973). 
También son muchas las publicaciones musicales que surgen en la segunda mitad del siglo XIX. Entre ellas podemos destacar «lberia musical y literaria" (1842) primer periódico de arte fundado por D. J. Espín y D. M. Soriano en el que la música ocupaba un importante apartado (M. Soriano, 1859, pag. 47); "La escena" Revista semanal de música, Madrid 1865; "El eco musical» Semanario de Literatura y Bellas Artes, La Coruña 1877; "El Boletín musical» Periódico artístico y literario, Madrid 1878; "La gaceta musical de Madrid, $1855 \ldots$

Pero en general, el compositor español se encontraba acorralado por una burguesía sin tradición musical y se veia encerrado en trabajos menores que constituian la única manera de ser tolerados. Baste recordar la ausencia casi absoluta de una vida sinfónica e incluso de música de cámara a lo largo de la centuria. La música de salón y la zarzuela eran los únicos campos que le estaban permitidos.

La única actividad que tenía un cierto ambiente en la sociedad española del XIX era el teatro lírico. Ello significaba, desde luego, la ópera italiana que deminó aplastantemente todo el siglo. Los intentos de crear una ópera nacional chocaron con una indiferencia social impenetrable. Por tanto, el compositor español hubo de refugiarse en la zarzuela y el género chico, que al menos contaba de partida con un público formado por la pequeña burguesía y clase popular y que con el tiempo, acabó por cautivar a todas las clases sociales con figuras como las de Bretón, Chapí, o Fernández Caballero. (C. Gómez, 1984, pag. 2031. En el campo de la zarzuela se obtuvieron logros, aunque estos músicos podrían haber dado mucho más juego en la ópera o terrenos casi inexistentes como el sinfonismo o la música de cámara.

La llegada del siglo XX sorprende a la vida musical española con una serie de problemas que arrastraba del siglo XIX — como acabamos de ver-, aunque figuras como Albeniz o Granados comenzaban a despuntar. Pero es un hecho, que, los avances que se producen en los primeros años del siglo fueron muy rápidos: La Sociedad Filarmónica de Madrid se crea en 1901, la Orquesta Sinfónica de Madrid en 1904 y la labor que en ella realizó E. Fernández Arbós hizo que los compositores españoles pudieran contar con instrumentos musicales de primera categoría, algo que con la precaria Sociedad de Conciertos no se hubiera podido conseguir; en 1915 la Orquesta Filarmónica de Madrid dirigida por B. Pérez Casas, cuya labor fue similar a la Sinfónica. Paralelamente, en Barcelona J. Lamote de Grignon creaba en 1910 la Orquesta Sinfónica. También la música coral conoció un renacimiento con la creación de coros estables en Bilbao, San Sebastián y Pamplona y posteriormente en Barcelona con el Orfeón Catalán. (T. Marco, 1983, pag. 21).

También es de reseñar, la labor que en este momento desarrollan los conservatorios, -especialmente los de Madrid y Barcelona-, que se convierten en centros musicales de gran vida. La mayoría de los músicos importantes del período fueron profesores en estos centros. Junto a ello, hemos de destacar la labor musicológica, que si en el siglo anterior habían desarrollado $\mathrm{H}$. Eslava 
y F. Pedrell, ahora es continuada por R. Mitjana, C. Rojo o D. Pujol entre otros. Todo ello junto a la crítica musical, que contribuyó en gran medida a aclarar y organizar la obra de sus contemporáneos entre los que destacamos a $\mathrm{C}$. de Roda, M. Salvador, A. Salazar, J. Turina... (J. Borrell, 1945, pag. 221).

\section{El movimiento romántico en la música española}

El Romanticismo tanto en su primera como segunda época, lo podemos considerar como un movimiento general que consigue apartarse del Clasicismo, abriendo un nuevo camino que afectó a todas las artes. El Romanticismo musical tiene dos corrientes diferenciadas: La producida en los estados germánicos, que se aparta de la realidad y tiene su origen en el "Sturm un Drang" (tormenta y tensión) de Goethe, Herder y Schiller; y el producido en Francia, impregnado del espíritu revolucionario y que a finales del siglo dará origen al Impresionismo, teniendo en C. Debussy (1862-1918) su mayor exponente (B. Traversoni, 1984, pag. 30).

Las características del Romanticismo musical se pueden sintetizar en: Un fuerte sentimiento nacional con el nacimiento del folklore, desarrollo de la canción popular y aparición de escuelas nacionales como la rusa, noruega, polaca, etc...; libertad y relación con los movimientos revolucionarios; desarrollo de la musicología y de la investigación histórico-musical; búsqueda de inspiración en ambientes exóticos, principalmente mundo árabe; relación entre las artes ... (G. Abraham, 1987).

El romanticismo español, sobre todo en música, no se puede enjuiciar ni valorar con la misma escala que se usa para el gran romanticísmo europeo. España desde el siglo XVIII, -si dejamos aparte algunas figuras destacadas y el florecimiento de un género de carácter nacional- no tiene compositores importantes, si exceptuamos los líricos, hasta Albeniz o Granados cerrando el XIX. Similar es escaso de la primera treintena del siglo $X X$, con varios nombres de primera línea como Falla, del Campo, Turina y Esplá, y otros secundarios como Arregui, Del Villar, Guervós, Manen... netamente románticos y casi desconocidos para el gran público, principalmente por el escaso apoyo y deficiente infraestructura de la música nacional.

Los músicos, en líneas generales, están sujetos en sus primeros tiempos al ambiente menor de lo que llamamos «música de salón». Esto e lo que diferencia fundamentalmente al romanticismo español del que se desarrolla en el resto de Europa. En todos los paises se hace este tipo de música, pero sobre la tradición del lied, la bagatela beethoveniana y las piezas de Schubert, se hace gran música en formas pequeñas. En Francia el lied se convierte en "melodía" o canción de concierto, mientras en Italia reina la canción sentimental. En España, con honrosas pero pocas excepciones, el nivel no se alcanza porque se carece de auténtica tradición.

Este problema junto a los que hemos visto anteriormente, van a ser una constante a la hora de plasmar las características generales que hemos des- 
crito para el Romanticismo y en concreto para en la que ahora nos vamos a detener: «Búsqueda de inspiración en ambientes exóticos y principalmente en el mundo árabe, en la música española durante la segunda mitad del siglo XIX y primera del XX".

La música, la pintura y la literatura son parcelas culturales que en este momento vuelven sus ojos en busca de inspiración, hacia un mundo oriental en general y norteafricano en el caso concreto de España. Estas influencias van a tener su plasmación en figuras como Galdos en literatura o Fortuny y Bertuchi en pintura. En música tenemos que hacer la distinción entre varios estilos: En un primer apartado estaría la Zarzuela y la música orquestal, en segundo las marchas moras, típicas del folklore levantino y en tercer y último lugar la música militar representada en himnos y marchas y a la que no haremos referencia por tener un carácter bélico y diferente a las anteriores.

Lo primero que hemos de señalar es que el reflejo de motivos árabes en estas obras, es básicamente un hecho artístico con un escaso o nulo, -en la mayoria de los casos-, trasfondo técnico. No encontramos, tras el análisis de una amplia muestra de piezas cuyo título o argumento pudieran sugerirnos una aproximación a un mundo exótico, ninguna unidad rítmica árabe fundamental como pueden ser el: Hazaj, Rámel, Thaquil primero y segundo o Majuri. Solo en algunas ocasiones modulaciones o intervalos como los de 2. ${ }^{a}$ aumentada nos podrían situar, solo de manera superficial en un ambiente oriental.

El compositor utiliza en la mayoría de los casos el motivo árabe, unicamente como pretexto ya que en ocasiones, no existe ninguna relación entre el encabezamiento de la obra y lo que a continuación se desarrolla en ella. El compositor se suele mover entre estereotipos orientales, que en la mayoría de los casos sirven unicamente para crear confusionismo. (Portadas número $1,2,3$ y 4 ). Dichos motivos solo se reflejan en las composiciones de forma superficial y de "oido" en el mejor de los casos.

Para comprender la producción musical española en este campo, es necesario tener una visión aunque muy ligera -como hemos señalado en otro apartado-, del contexto histórico y social del músico español. La falta de tecnicismo a la hora de abordar este tema, no hemos de achacarlo unicamente a que la preparación de nuestros compositores fuera baja, sino también a otros problemas que exceden la individualidad de músico, lo someten a su marco social y a lo que éste le demanda. Son muy gráficas unas frases de don $\mathrm{H}$. Eslava en 1871 en su tratado "Escuela de composición»:

«Es necesario tener presente que además de las piezas, que en general tienen una estructura correcta... suelen presentar algunas veces composiciones de una estructura caprichosa y que llevan títulos, que por sí solos absuelven a los autores de todas las incorrecciones y aún desatinos que hayan podido cometer. Tales son algunas piezas instrumentales... con los títulos de fantasía, miscelania, delirios, recuerdos de baños... en 
cuyas obras por mero capricho o por ignorancla encubierta, tan procedido libremente respecto a la estructura, en otras se falta a ello. En efecto, que censura puede haber contra la incorrección de estructura de una pieza que expresa el sueño, delirio o locura. Sepase, pues, que aunque en algunas obras de este género suelen respetarse y observarse los principios del arte respecto a su estructura, en otras se falta a ellos; y en ese caso no hay lugar a exámen alguno, debiendo ser calificadas, si no es de malas, por lo menos de románticas».

El fragmento, creemos que es lo suficientemente elocuente por sí solo y más si tenemos en cuenta que en esos momentos en Europa se asistía a la segunda oleada romántica, con figuras como las de Brams, triunfaba el nacionalismo y se imponía la figura de Wagner.

Pese a todo, hemos intentado hacer un análisis de algunas obras o fragmentos de ellas, que desde un punto de vista armónico podían tener algún contacto y recreación del mundo árabe.

\section{La Festa del poble de Fco. Alonso}

Pasodoble morisco escrito por el maestro Fco. Alonso con motivo de las Hogueras de San Juan de Alicante y estrenado en las del año 1934, por la banda de «moros» Nueva Iris de Alcoy. (Bilbao, Unión Musical Española, S.a. 7 pag.).

Rítmicamente la pieza esta escrita en compás de 2/4. Los tiempos utilizados son dos: El Pasodoble con el que se inicia la obra y el Bolero a partir del compás número 52.

Pasodoble

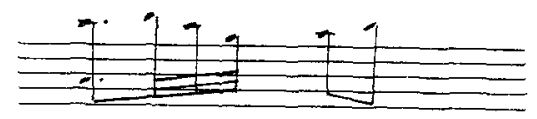

Bolero

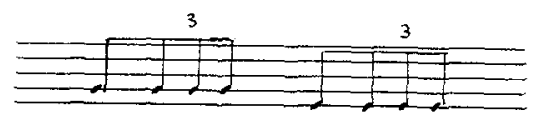

Dentro de los rítmos árabes fundamentales de $2 / 4$ estaría más cerca de la fórmula del "Thaquil aurval» $\operatorname{liger}_{4}, \Gamma, \mid \Gamma !$ que la del "Hazaj»

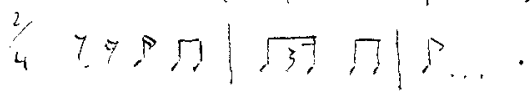

En toda la obra aparecen motivos característicos de los timbales y atabales, así como los motivos $\{.7$, 
que son fórmulas rítmicas construidas a base de notas de adorno para destacar los melismas propios de esta música y destinados a instrumentos de viento.

El modo como es característico en la música árabe es menor comenzando por la dominante. Esta pieza esta escrita en Sol menor. Toda la introducción se forma por una pedal extensa sobre la dominante (20 compases). En el compás número 21 se inicia la melodía con los típicos floreos. Utiliza dentro de la escala de Sol menor en unas ocasiones la armónica y en otras la natural. No es de estrañar la utilización de estos tipos de escala natural ya que Al-Fārābĩ e lbn Sīnā, se basan en el sistema griego, clasificando las escalas en grupos fuertes, en las que se incluyen las de género diatónico y en grupos débiles que comprenden las escalas de los géneros cromáticos y enạrmónicos. En el sistema tonal, que es el empleado por los músicos españoles, los géneros más utilizados son estos dos, con inversiones modales como son habituales en la construcción de la música tonal.

Ya hemos dicho anteriormente que la línea melódica se inicia en el compás número 21. En ella aparecen numerosas notas de adorno, al igual que intervalos de 2 a $^{\text {a }}$ aumentada y trinos ejecutados también en $2 .^{a}$ aumentada, siendo en estos donde se acusa más el sentido melismático típico de la música árabe. Otra de las características de esta música que encontramos en la melodía, la constituye la preferencia por los intervalos pequeños y conjuntos, ya que prefieren el evitar saltos grandes (principalmente pensando en la voz), y también las sucesiones melódicas de carácter ascendentes. También encontramos algunos cromatismos propios de la música árabe y de la oriental en general:

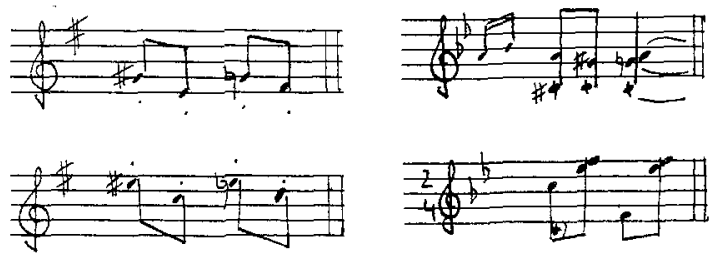

Estructuramente la obra posee dos temas independientes y se divide en:

- Estribillo.

- Parte instrumental.

- Tema A.

- Desarrollo del tema A.

- Estribillo introductorio.

- Tema B.

Estribillo instrumental.

Repetición íntegra del tema B.

El estribillo instrumental se inicia con una pedal de dominante que abarca 20 compases. De esos compases, 12 corresponden al ututti» de la orquesta o banda, mientras que a partir del compás número 12 comienza un pequeño diseño musical que por su carácter se asociaría a los instrumentos de vien- 
to y por tesitura podría ser el clarinete. Estos diseños de carácter eminentemente instrumental, se alternan con otros puramente rítmicos destinados para instrumentos de percusión como los timbales y atabales.

En el compás número 21 comienza el tema A. También esta escrito en Sol menor con los tipos de escalas que ya hemos mencionado. Abarca 32 compases y de ellos 16 son los que constituyen el tema en sí, siendo los otros 16 la repetición del mismo tema, con alguna ligera modificación a partir del compás número 25.

A continuación viene un desarrollo por imitación, tomados ritmicamente de los dos primeros compases del inicio del tema $A$.

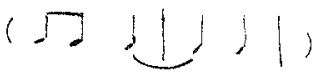

Este desarrollo esta constituido por 13 compases de los cuales los 4 primeros se repiten nuevamente, continuando después con una imitación rítmica.

Después de este primer tema y desarrollo, aparece de nuevo el estribillo instrumental del comienzo de la obra, nuevamente sobre una dominante prolongada que abarca 14 compases, uno más que el estribillo primero. El motivo de añadir este compás es el de preparar ampliamente el tema B. Justo estos dos compases añadidos proporcionan mayor énfasis a la entrada del segundo tema o tema B en la tomalidad de Sol Mayor.

El tema $\mathrm{B}$ contiene 32 compases al igual que el $\mathrm{A}$, de los cuales los 16 primeros constituyen la exposición en sí del tema y el resto con una ligera variante, son la repetición del primero pero en octava alta y con una variante del último período de esos 16 compases, es decir últimos 8 compases, destinados exclusivamente a preparar el final.

A continuación una nueva parte instrumental que conserva el ritmo pero no la tonalidad ya que por una modulación por $3 .^{\text {as }}$ se expone en el tono de Si menor. Seguidamente el mismo motivo rítmico se transporta al tono de $R e$ Mayor y dominante del tono principal de Sol Mayor. El motivo de que se inicie esta parte en Si menor, es porque el tema B termina en la tónica de Sol Mayor no era conveniente insistir demasiado en dicha tonalidad. Por otra parte este fragmento de estribillo instrumental contiene una pedal superior de dominante que abarca 6 compases y que sirve para preparar nuevamente la entrada del tema B íntegramente, es decir los 32 compases que lo configuran y terminando así la obra.

\section{La cautiva de Fco. Alonso}

Canción morisca, Letra de Jerónimo de la Cruz, Madrid Unión Musical Española (s.a. 1944, 4 pág.). 
La escala en modo menor (escala eólica) y su ritmo repetitivo son dos de las características que asemejan esta partitura a la música árabe. En concreto, el ritmo repetitivo que genera el primer tema es un ritmo «Hazaj»

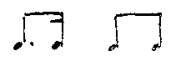

que esta al comienzo del tema $\mathrm{A}$ y $\mathrm{B}$.

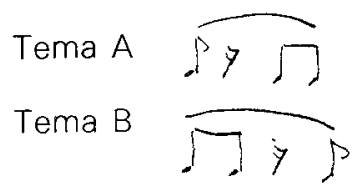

Hay abundantes notas de adorno producidas por floreo.

Su estructura se divide entre partes:

1. Introducción rítmica de 4 compases.

II. Tema A con 32 compases formados por períodos de 8 compases.

- Los 16 primeros constituyen la exposición del tema primero y los restantes son la repetición de éste nuevamente, con una variación en la última frase del último período.

- Desarrollo: Constituido a base del motivo inicial

$$
6, \sqrt{7}
$$

abarca 10 compases.

- Reesposición parcial del tema, solo sus 16 últimos compases. Coincidiendo con el final del tema que aparece en la melodía, encontramos un cromatismo que abarca 4 compases, interpretados por instrumentos (en este caso el piano) también característico de la música a la que nos estamos refiriendo.

III. Tema B. Si el primer tema se encontraba escrito en Si Menor modula aquí a Si Mayor. Abarca 49 compases de los cuales 32 constituyen ert esencial el tema $B$, siendo el resto una especie de coda.

El final del tema B interpretado por la voz, coincide con en inicio de este mismo tema, interpretado por la orquesta o los instrumentos. Los últimos compases de la obra contienen una pedal superior de dominante que interpreta la voz. Mientras que en la orquesta o instrumentos aparece el motivo que da origen al tema $A$.

\section{El moro muza de R. Chapí}

Ensayo cómico de un drama lírico en un acto, en prosa y verso original de Jaques Federico, música del maestro R. Chapí. Adm. Lírico Dramático 1894. Extreno teatral, Eslava 31 de octubre de 1894. 
La partitura analizada consiste en la «Romanza morisca» de dicha zarzuela para voz de soprano. Estructuralmente se divide en siete partes. En la primera o introducción, destacan varios giros melismáticos en los primeros compases (fa-sol-fa, sol-la-sol) y la repetición insistente de algunos de ellos:

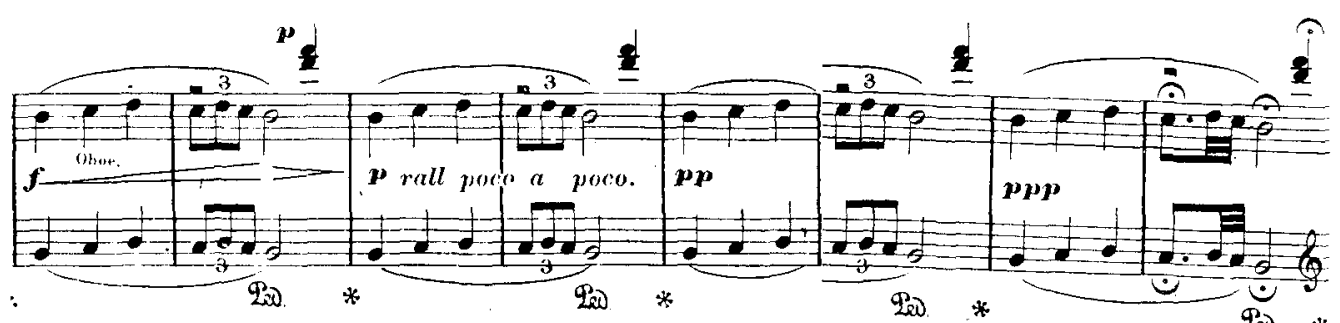

El acompañamiento instrumental se compone de instrumentos de viento esencialmente (trompas, flautas, trompetas, oboe y clarinete más fagot) y de madera.

En el segundo período (a partir del compás número 21) se inicia propiamente la melodía. En ella se acusa con mayor insistencia el sustrato melismático típico de la música árabe. (la-si-la-sol). La primera célula melódica esta compuesta por 8 compases, que a su vez constituyen dos frases cada una de 4 compases, siendo la segunda una repetición exacta de la primera. Lo mismo sucede en el período siguiente en el que siguen apareciendo los giros melismáticos más acentuados. El tercer período con las mismas características es una reproducción exacta del primero pero transportado a una distancia de tercera menor. En los dos períodos siguientes, la insistencia melódica acentua más la monotomía que encontramos en la música árabe. Igualmente el $2{ }^{\circ}$ período es una repetición exacta del primero. El $6 .^{\circ}$ período es repetición de la introducción, aunque aquí se introduce la voz y en beneficio de ésta se prescinde de los giros ornamentales. El 7. ${ }^{\circ}$ y último período reproduce de nuevo un fragmento de la introducción. (compases número 9 al 16).

En resumen, las posibles concordancias entre ambas músicas son: La estructuración de los períodos con repeticiones insistentes; la tonalidad general en la que se desarrolla la pieza que es un modo menor y la abundante utilización de $2 .{ }^{\text {as }}$ aumentadas y melismas.

A nivel compositivo hemos de señalar, que aún habiendo utilizado muy discretamente la modulación existe una gran pobreza inspirativa en el conjunto de la obra.

\section{Beny de R. Adua}

Marcha mora para piano, compuesta por el maestro R. Aduá. Musical Emporium, Barcelona. 
Lo más acusado en esta marcha mora, es precisamente las características propia de la marcha, con unas fórmulas ritmicas muy definidas:

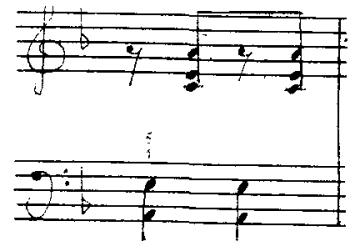

y que se conservan arraigadas en las fiestas de "Moros y Cristianos" del Levante español.

También en esta ocasión se utiliza un modo menor, con el empleo indistintamente del $6 .^{\circ}$ grado como sensible o perteneciente a la escala natural. Aparecen algunos ornamentos entremezclados, producidos por notas de floreo a nivel armónico.

El autor suponemos que como una concesión al público, y para acentuar la brillantez de la partitura, modula en la última parte hacia un modo mayor (Re Mayor), para terminar en un "fortissimo".

Estilisticamente volvemos a encontrar giros melismáticos u ornamentaies propios de la música árabe, así como la insistencia en la repetición melódica. Pero todo ello siempre en un nivel muy superficial de imitación externa.

Técnicamente podemos observar, que al utilizar preferentemente el «modo menor" de nuestro sistema tonal, nos permite por una parte, la aparición de giros característicos de $2 .^{\circ}$ aumentada, propios de la escala armónica como de la escala natural, con lo cual, al utiiizar la alteración del $7 .^{\circ}$ grado de la escala natural como el de la armónica, estamos tomando la ambientación de la escala andaluza (mi-fa-fa \#-sol-sol \#-la-si-do-re-mi-).

Estructuramente no tiene relación con la música árabe. Sus características son las propias de una marcha occidental, tanto formalmente (fraseos, períodos...) como por su sistema tonal de modulaciones.

\section{Marcha morisca de R. Chapí}

Marcha morisca para piano del maestro R. Chapí. Sociedad de Autores Españoles, Madrid, 1908.

La obra parte de una célula (1) que encontramos en el primer compás de la Introducción (Allegro moderato). Dicha célula va a aparecer a lo largo de toda la obra, unas veces con movimiento directo y otras por movimiento contrario: 


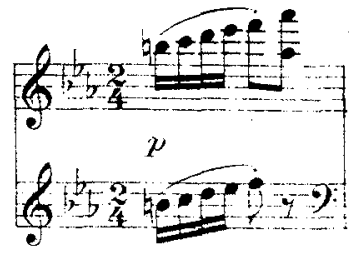

Estructuramente la obra se divide en dos partes claramente diferenciadas; la primera como ya hemos dicho, esta compuesta por una Introducción (Allegro moderato) y la segunda por una Danza (Allegro).

En la Introducción encontramos elementos que después se van a repetir en la Danza, como son las 4 semicorcheas del primer compás (representado en la parte superior) o las 4 semicorcheas de los compases número 8 y 9 con un dibujo melódico característico:

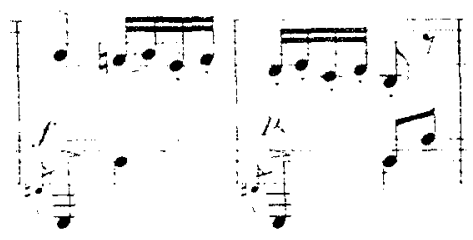

También se encuentran en esta introducción, pequeños diseños que como el autor indica imitan los sonidos del tamboril. Aparecen por primera vez en el compás número 2 y sirven de acompañamiento en un gran parte de la lntroducción:

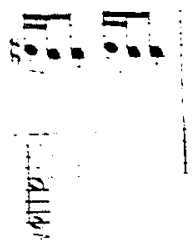

Está escrita en un modo menor (Do menor) y en ella aparecen intervalos característicos de $2 .^{a}$ aumentada (la -si), así como giros repetitivos. La unidad estructural de la obra comprende períodos de 8 compases. en los que por general la primera frase ( 4 compases) es imitada exactamente en la frase siguiente.

La Danza (Allegro) esta escrita en compás de $3 / 8$ y para ser marcada al tiempo. Aparece aquí la misma estructura en períodos, en los que la $2 .^{a}$ frase es una repetición exacta de la $1 .^{a}$. Hay 3 elementos o diseños que configuran la estructura de esta 2. ${ }^{a}$ parte: El $1 .^{\circ}$ de ellos aparece en el primer compás, partiendo de un grado diferente y de forma invertida. Este mismo diseño ligeramente modificado y por imitación, se encuentra como elemento $2 .^{\circ}$ entre 
los compases número 8 y 9 . Por último el $3 .^{\text {er }}$ elemento se ha tomado de los compases número 8-9 de la Introducción.

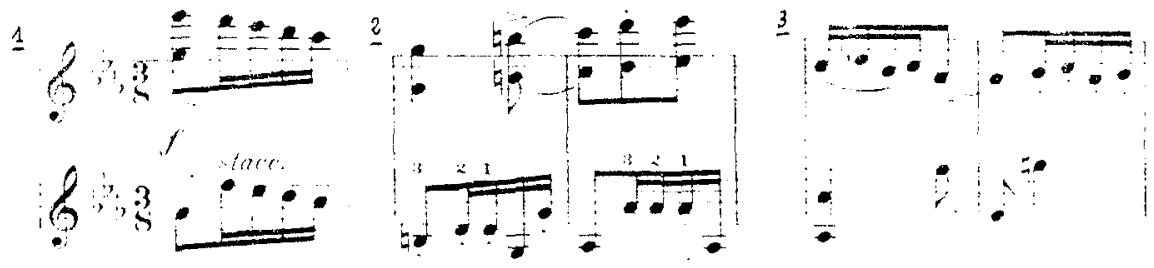

En los compases del número 15 al 22, de la Danza, se muestran dos posibles diseños a elegir por el interprete. Ambos diseños no tienen ninguna relación con la música norte-africana sino que están ligados a la música tonal y destinado al virtuosismo dentro de sus esquemas.

Los compases siguientes son la preparación del final, en ellos aparecen nuevamente la célula ritmíco-melódica que sirvió para estructurar la obra.

\section{Moraima de G. Espinosa}

"Capricho característico". Transcripción para violín y piano de E. Boccalari. Buenos Aires, Ricordi (s.a.).

Muy pocas características de la música oriental encontramos en esta partitura a excepción de algunos giros que encontramos en los compases número 52-53, o algunas notas de adorno producidas como en este caso por floreos:

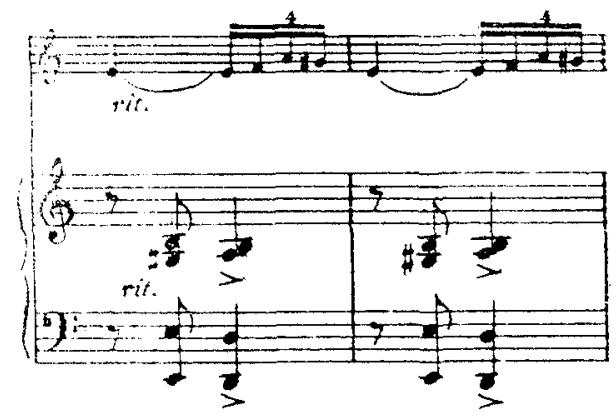

El tono inicial de exposición del primer tema es La menor. El autor utiliza tanto la escala natural como la armónica. En el segundo tema pasa a utilizar un modo mayor (La Mayor).

La célula inicial que origina el tema primero es un motivo breve que podríamos considerar con características orientales tales como la utilización de grados conjuntos y el sentido ascendente de la melodía que encontramos especialmente en la introducción. 


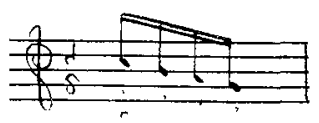

La estructura se compondría de cuatro partes:

1. a Introducción: Constituida por los 20 primeros compases.

2. ${ }^{\circ}$ Tema A: Formado por 34 compases. En ella tendríamos una primera exposición del tema, que va a enlazar con el relativo mayor (Do mayor) y una reexposición del tema ampliado para concluir.

Como puente o enlace del final de la reexposición del tema con el inicio del tema $B$, hay en el piano un cromatismo ascendente.

3. ${ }^{\circ}$ Tema B: La célula inicial del tema B está sacada por inversión rítmicaa de uno de los ritmoś que aparecen en el tema A. En el tema B en La Mayor aparecen frecuentes progresiones melódicas aśl como algunos fragmentos pertenecientes al desarrollo del tema A. (C.45-47).

4. ${ }^{\circ}$ Reexposición final del tema $A$, pero ampliado a manera de conclusión final (c. 128 al final). En los compases 135 a 137 aparece la $6 .^{a}$ napolitana. Y los compases 139- al 144 están formados por una coda en la que en el bajo aparece la célula rítmica que origina el tema A escrita por ampliación.

\section{En la Alhambra de T. Bretón}

Serenata para piano del maestro Tomás Bretón. Madrid, Unión Musical Española (s.a.).

Esta obra, contiene características muy acusadas de la música andalusí, tanto por los giros de la melodía (2. as menores, ornamentaciones...) como por la utilización de la escala andaluza. Así podemos ver, que aunque la obra está situada en la tonalidad de La menor, no cabe duda que la aparición del Fa natural y el Fa \# (sostenido) y el Sol natural y Sol(\#)(sostenido), nos situa en la escala andaluza como referente. También aparece el Si (bemol) que aunque no es propio de esta escala si lo es de la música andaluza en general.

La estructura corresponde a la forma «Rondó» en la que se alterna la copla y el estribillo. Contiene dos secciones perfectamente definidas: En la primera se alterna el estribillo coplas diferentes musicalmente y la segunda sección es la repetición de la primera con ligeras modificaciones en el final a modo de conclusión.

\section{Fantasía morisca de R. Chapí}

"Fantasía morisca para orquesta» (Final), para el maestro R. Chapí y arreglada para piano. La correspondencia musical, Madrid.

En el movimiento Final de la Fantasía morisca, (compendio de las anteriores), no encontramos muchos rasgos que podamos asociar con la música oriental. Solo en el inicio vemos algunos rasgos comunes como son la ornamentación muy acusada que observamos en el "moderato» inicial. En este caso, aunque utiliza el modo mayor con objeto de imprimirle una mayor bri- 
llantez, al final sí utiliza sin embargo, el $7 .^{\circ}$ grado de la escala como tal grado y no como sensible, lo que aumenta la incidencia sobre los rasgos señalados.

El resto de la obra no contiene nada que pueda identificarse con la música a la que estamos haciendo referencia, pues se limita a una serie de dibujos puramente virtuosísticos (ni siquiera melódicos), que nos hacen pensar más en un ejercicio escolástico que en una obra destinada a orquesta.

\section{Serenata árabe de Zielnski}

Se divide en dos secciones claramente diterencladas. tn la primera se hace la exposición de un tema que es repetido parcialmente en la segunda (únicamente los 5 primeros compases que siguen a la Introducción).

La influencia oriental se plasma especialmente en la insistencia en unos giros determinados que se repiten machaconamente en distintos grados de la escala. Muchos de estos giros nos hacen pensar en ciertas partes instrumentales que de manera virtuosística se interpretarían a la guitarra.

Como en otras ocasiones, el autor ha elegido la tonalidad de La menor para desenvolver la obra. Resulta curioso la introducción de un único compás de $3 / 4$ (resto en 6/8), un compás antes del inicio de la segunda sección. Nos podría hacer pensar en la combinación 6/8 más 3/4 propia de la petenera. 


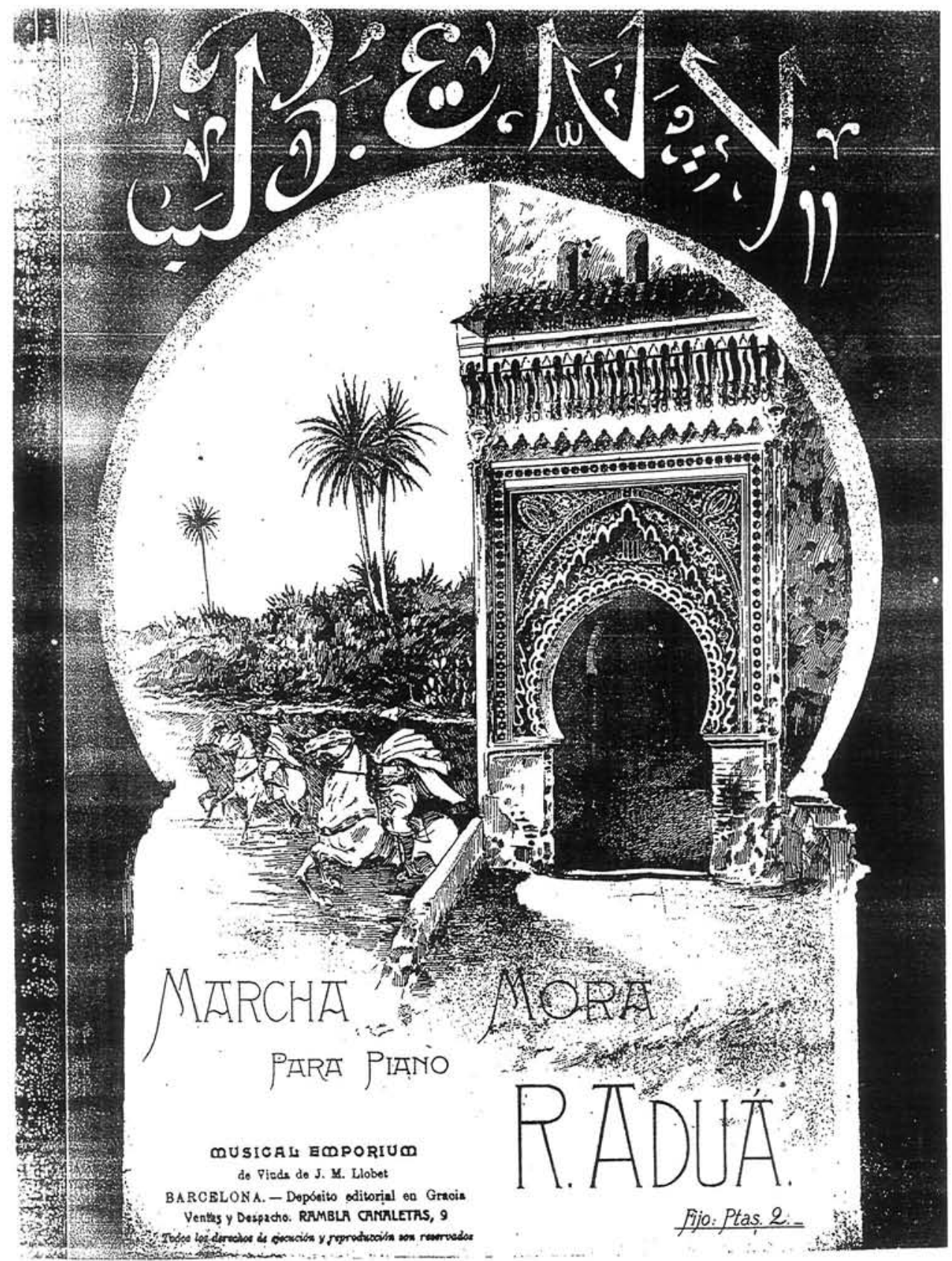


WEALIUN DE LA UENIALAKIISIA LA ARGENTINITA
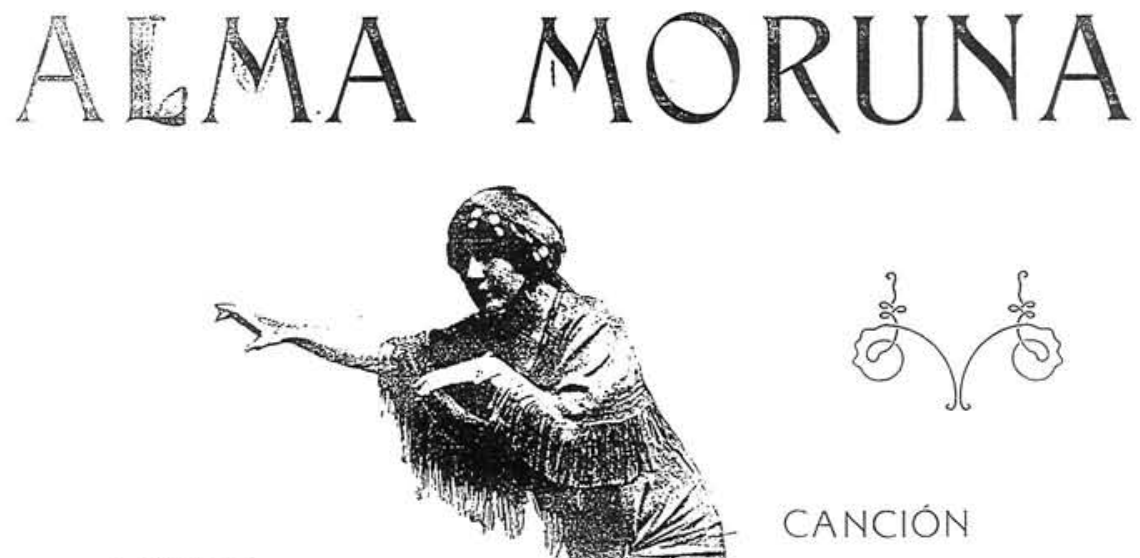

1 IRA:

CANCION

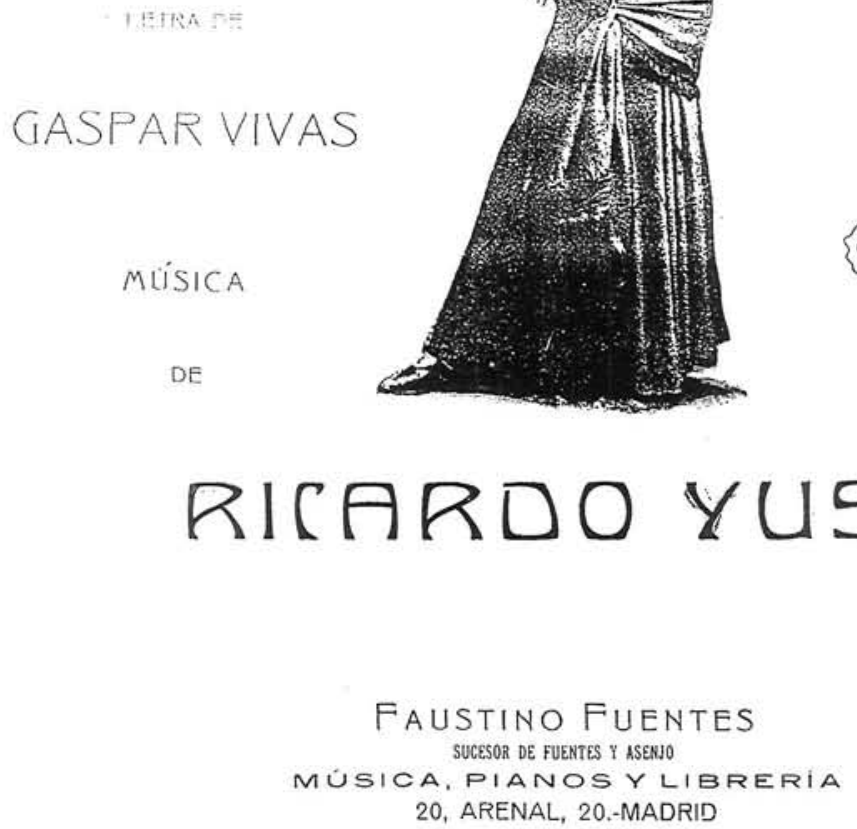

ORIENTAL

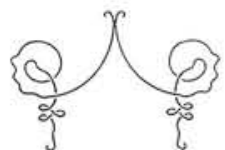

PR. 2 PrAS, FIJO

20, ARENAL, 20.-MADRID 


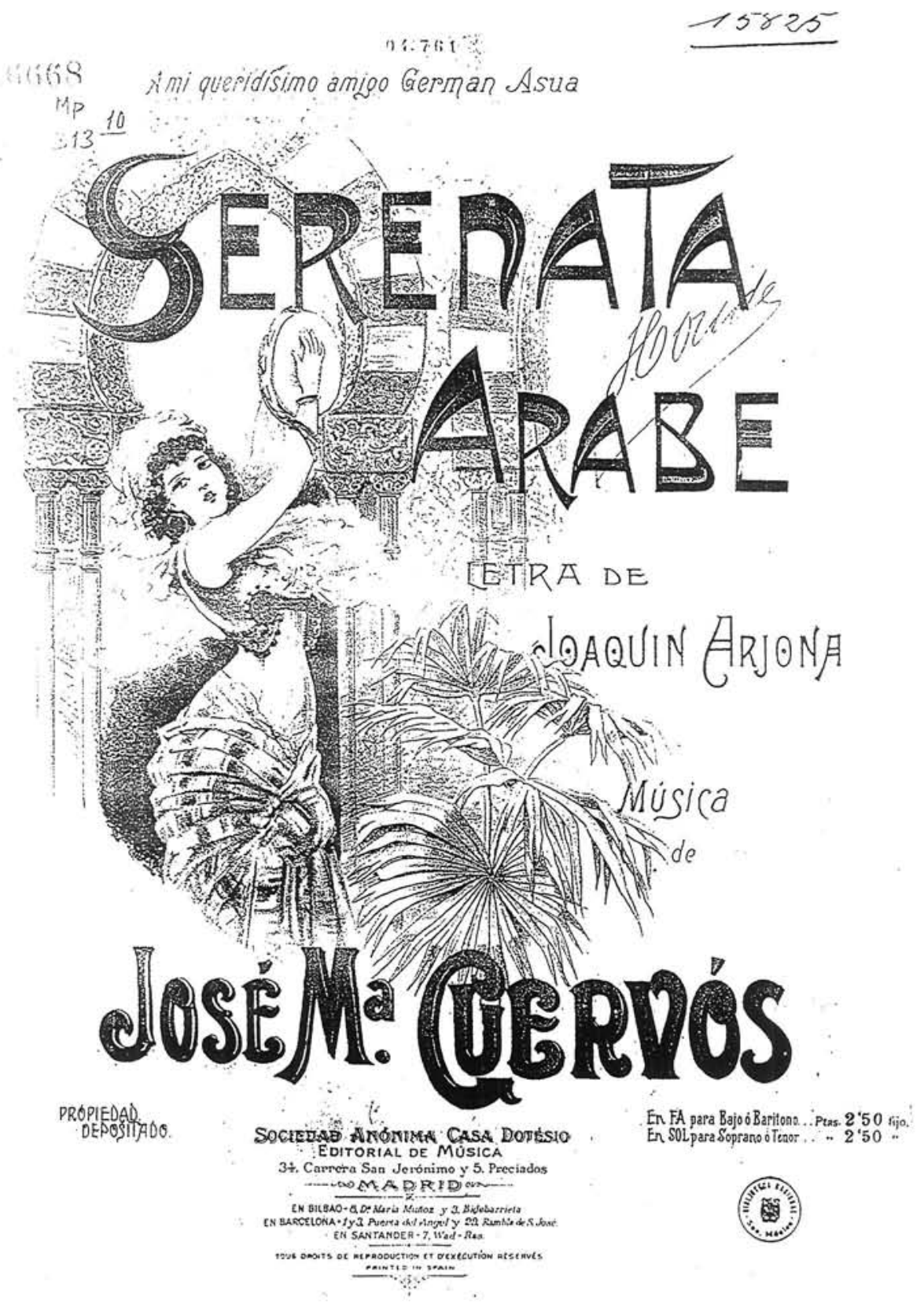




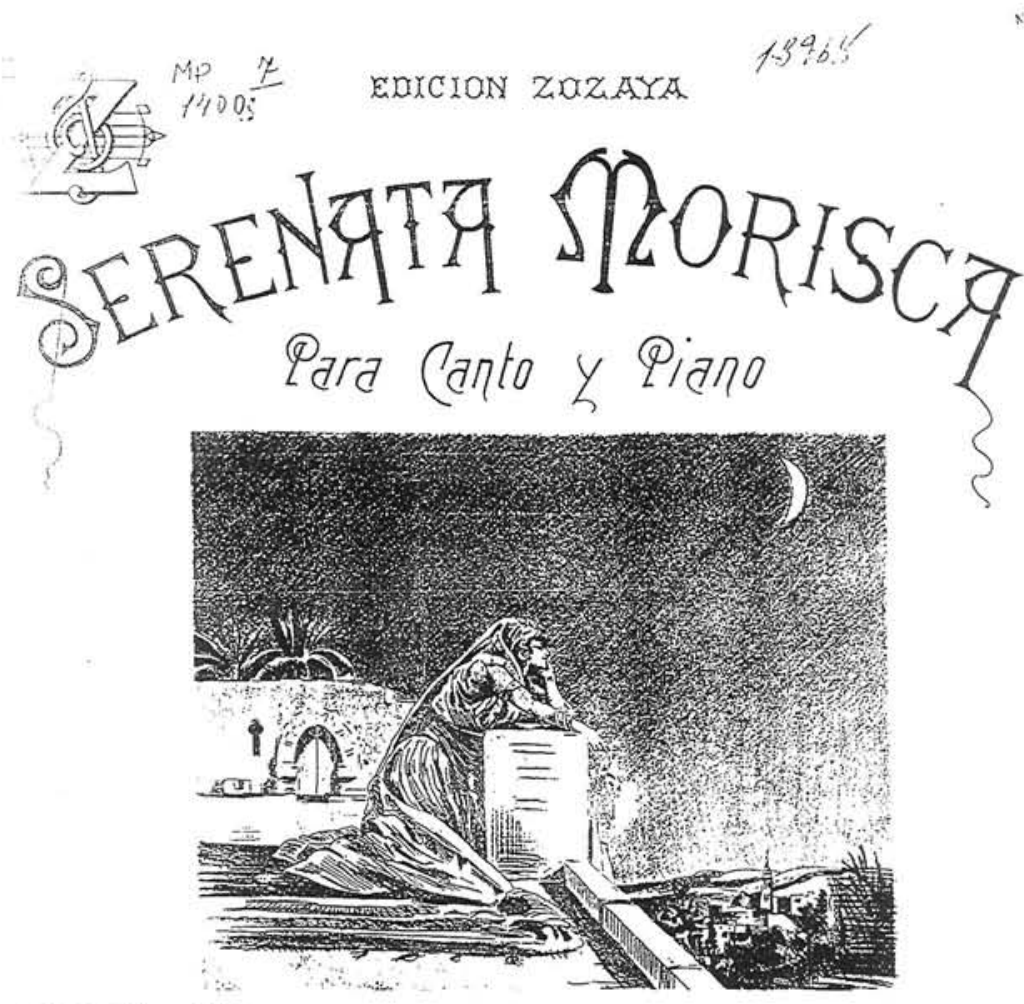

LETRA DE

Q.BONET.

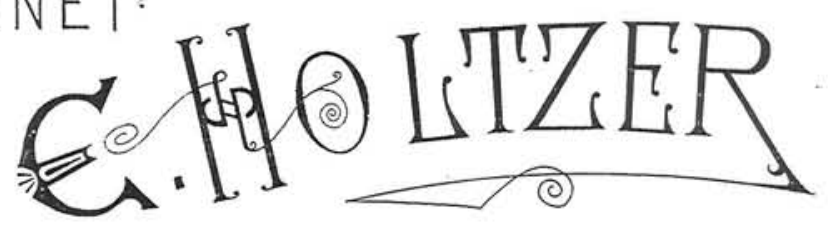

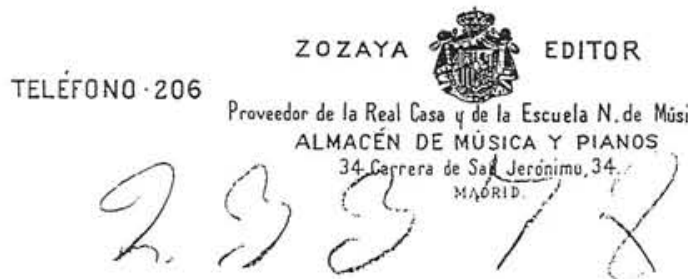

PROPIEDAD PARA TODOS LOS PAISES 


\section{BIBLIOGRAFIA}

ABRAHAM, G. Historia universal de la música. TAURUS EDICIONES, Madrid, 1987.

BORRELL VIDAL, J. Sesenta años de música (1876-1936). Ed. Donat, Madrid, 1845.

CARR, R. España: de la Restauración a la democracia, 1875-1980. Ed. ARIEL, Barcelona, 1983.

FALLA, M. de Escritos sobre música y músicos. Col. Austral, ESPASA-CALPE, n. ${ }^{\circ}$ 950. Madrid, 1972.

FERNÁNDEZ-CID, A. La música española del s. XX. Fund. March, Madrid, 1973.

GÓMEZ AMAT, C. Historia de la música española, s. XIX. Alianza Editorial, T. 5, Madrid, 1984.

JARGY, S. La musique arabe. ¿Que sais-je? Ed. Puf. París, 1971.

MARCO, T. Historia de la música española, s. XX. Alianza Editorial, T. 6, Madrid, 1983.

MENÉNDEZ PIDAL, R. Poesía árabe y poesía europea. Col. Austral, ESPASA-CALPE, n. ${ }^{\circ}$ 190, Madrid, 1973.

MILO, D. "Le musical et le social, XVIII ${ }^{e} X \mid X^{e}$ siècle». Annales ESC, janvier-février 1987, n. ${ }^{\circ} 1$, pp. $27-40$.

RIBERA Y TÁRRAGO, J. La música árabe y su influencia en la española. Ed. Mayo de Oro, Madrid, 1985.

SORIANO FUERTES, M. Música árabe-española. Barcelona, J. Ulivers, Impresores de S. M., 1853.

SORIANO FUERTES, M. H. ${ }^{\circ}$ de la música española desde la venida de los fenicios hasta el año 1850 . BarceIona, J. Olivers, Impresores de S. M., 1859.

TRAVERSONI, B. La ciencia, el pensamiento y el arte en el siglo XIX. Cuadernos de estudio, Ed. C. K. n. ${ }^{\circ}$ 28, Madrid, 1984.

VERNET, J. La ciencia en Al-Andalus. Biblioteca de la Cultura Andaluza. Sevilla, 1986.

VIGUERA, M. ${ }^{\circ}$ J. "El N. de África en la música europea del s, XIX». Rev. Awraq, vol. I, 1987.

ZAUAS, R. de Origenes de la ópera. Aportaciones arábigo-andaluzas. Ed. Alpuerto, Madrid, 1982. 